DE

M E D I C I N A

T R O P I C A L

$\mathrm{DE}$

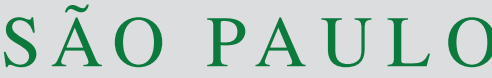

JOURNAL OF THE SÃO PAULO INSTITUTE OF TROPICAL MEDICINE

${ }^{1}$ Ministry of Public Health, National Institute of Health, Department of Medical Sciences, Nonthaburi, Thailand

${ }^{2}$ Mae Sot Hospital, Department of Medical Technology, Tak, Thailand

${ }^{3}$ Hokkaido University, Research Center for Zoonosis Control, Division of Bioresources, Sapporo, Japan

${ }^{4}$ Hokkaido University, Global Institution for Collaborative Research and Education, Global Station for Zoonosis Control,

Sapporo, Japan

Correspondence to: Benjawan Phetsuksiri Ministry of Public Health, National Institute of Health, Department of Medical Sciences, $88 / 7$ Tiwanond Rd, 11000, Muang, Nonthaburi, Thailand

Tel: + 6625801567

Fax: +6629659700

E-mail: benjapsk@ health.moph.go.th

Received: 6 January 2020

Accepted: 27 April 2020

\section{The performance of an in-house loop-mediated isothermal amplification for the rapid detection of Mycobacterium tuberculosis in sputum samples in comparison with Xpert MTB/RIF, microscopy and culture}

\author{
Benjawan Phetsuksiri ${ }^{\circledR}$, Wiphat Klayut ${ }^{1}$, Janisara Rudeeaneksin ${ }^{1}$, Sopa \\ Srisungngam ${ }^{1}$, Supranee Bunchoo ${ }^{1}$, Sarawut Toonkomdang ${ }^{2}$, Thanee \\ Wongchai $^{2}$, Chie Nakajima ${ }^{3,4}$, Yasuhiko Suzuki ${ }^{3,4}$
}

\section{ABSTRACT}

Simple, low-cost and effective diagnostic tests for tuberculosis (TB) are needed especially in TB-high burden settings. The present study evaluated the performance of an in-house loop-mediated isothermal amplification (LAMP) for diagnosing TB by comparing it to Xpert MTB/RIF, microscopy and culture. In Thailand, a total of 204 excess sputum samples volume after the processing of cultures were used for Mycobacterium tuberculosis (MTB) detection by Xpert MTB/RIF and LAMP. Based on culture results as the gold standard, the overall sensitivity of LAMP and Xpert MTB/RIF were 82.1\% (126/153; 95\% confidential interval [CI]: $75.4-88.98 \%)$ and $86.9 \%(133 / 153 ; 95 \%$ CI: $80.5-90.8 \%)$ respectively, and the specificity of both tests was $100 \%$ (51/51; $95 \%$ CI: 93.0-100.0\%). In comparison with Xpert MTB/RIF, the sensitivity and specificity of LAMP were $94.7 \%(126 / 133 ; 95 \%$ CI: $89.5-97.9 \%)$, and $100.0 \%$ (73/73; 95\% CI: 94.9-100.0\%), respectively. The average threshold cycle $(\mathrm{Ct})$ of Xpert MTB/RIF detection for positive and negative LAMP results was statistically different, of 18.4 and 27.0, respectively $(p<0.05)$. In comparison with the acid-fast staining technique, and analyzing LAMP and Xpert MTB/RIF in smear-negative/ culture-positive specimens, there was an increase of the detection rate by $47.7 \%(21 / 44)$ and $54.6 \%$ (24/44). The diagnostic sensitivity and specificity of LAMP appeared to be comparable to those of Xpert MTB/RIF. We claim that this LAMP has potential to provide a sensitive diagnostic test for the rapid TB diagnosis. It allowed a fast detection of MTB before the cultures and it could be used in resource-limited laboratory settings.

KEYWORDS: LAMP. Tuberculosis. Diagnosis. Sputum.

\title{
INTRODUCTION
}

Tuberculosis (TB) remains a global health problem. Recently, in 2018, there were an estimated 10 million new TB cases and 1.4 million deaths worldwide ${ }^{1}$. Rapid and accurate diagnostic tests are required to notify TB cases. Even though microscopy for acid-fast bacilli is easy, fast and inexpensive to perform, this method has a low sensitivity and cannot distinguish between Mycobacterium tuberculosis (MTB) and non-tuberculous mycobacteria (NTM) ${ }^{2}$. For this reason, several tools based on molecular techniques to detect MTB nucleic acids directly from clinical specimens were developed. In 2010, the World Health Organization (WHO) endorsed Xpert MTB/RIF (Cepheid, Sunnyvale, CA, USA), an automated realtime $\mathrm{PCR}^{3,4}$. This molecular technique can simultaneously detect MTB DNA and 
the resistance to rifampicin, the most effective drug used for TB therapy, based on the detection of the $r p o B$ gene and its mutations, which confer resistance to rifampicin. GeneXpert machines and cartridges have been distributed and available in many regions in both, high and low-income countries. It has rapidly become widespread as a molecular point-of-care test (POCT) and provided a new approach for the rapid TB diagnosis. However, the economical technique is still required in many resource-limited countries with a high-TB burden like Thailand, and others, since GeneXpert instruments require high maintenance service charges and cartridges are expensive for lowincome areas. A quite effective, but inexpensive screening test for MTB detection might reduce the costs from using Xpert MTB/RIF tests in many TB cases. Loop-mediated isothermal amplification (LAMP), a novel nucleic acid amplification test was developed ${ }^{5,6}$ and has several advantages for the rapid MTB detection. It provides a highly sensitive and specific detection at low costs and no need of sophisticated machines. A commercial product to detect MTB based on the LAMP technique has been developed by Eiken Chemical Company (Tokyo, Japan) ${ }^{7}$ and now is available in more countries. Previously, Pandey et al. ${ }^{8}$ proposed the use of an in-house LAMP to detect MTB directly from processed sputum samples. In comparison with a gold standard culture technique, this in-house LAMP showed high sensitivity (100.0\%) and specificity (94.2\%). This could be applied for the rapid confirmation of MTB growth in culture media and we reported its high sensitivity and specificity ${ }^{9}$. However, the diagnostic performance data of this in-house LAMP in this setting is limited. Here, we evaluated the performance of an in-house LAMP for the rapid detection of MTB in processed sputum samples by comparing it to Xpert MTB/RIF and mycobacterial culture. The study was carried out in Thailand, a TB-endemic setting, where the use of a LAMP technique would be appropriate.

\section{MATERIALS AND METHODS}

\section{Clinical specimens and sample processing}

This study was approved by the Ethics Committee of Mae Sot Hospital. From April to September 2018, sputum specimens were consecutively obtained from patients with symptoms compatible with TB. The clinical specimens were processed according to the routine mycobacteria laboratory procedure at Mae Sot Hospital, Tak province. After receipt, sputum samples were checked and decontaminated using the sodium hydroxide-N-acetyl-L-cysteine (NaOH-NALC) method ${ }^{10}$. Briefly, an equal volume of the decontamination solution together with digestion agents were added to each sputum sample in a 50-mL centrifuge tube. After remaining at room temperature for $15 \mathrm{~min}$, the mixture was vortexed and subsequently neutralized with a phosphate-buffered saline (PBS; pH 6.8). Then, the mixture was centrifuged at $3,000 \times \mathrm{g}$ for $15 \mathrm{~min}$ at $4{ }^{\circ} \mathrm{C}$ and the supernatant was discarded. The pellets were resuspended using $2 \mathrm{~mL}$ of PBS. The bacterial culture was then conducted in both, solid (Löwenstein-Jensen; LJ) and liquid medium (Mycobacteria Growth Indicator Tube; MGIT; Becton Dickinson, San Diego, CA, USA) by inoculating $\sim 100 \mu \mathrm{L}$ and $500 \mu \mathrm{L}$ of the cell suspension to LJ and MGIT culture media, respectively. Mycobacterial growth was observed periodically. Microscopic examination for acid-fast bacilli (AFB) was performed in positive cultures and the growth of bacteria was confirmed for MTB by a rapid immunochromatographic identification test (SD BIOLINE TB Ag MPT64 Rapid kit; Standard Diagnostics, Korea) according to the manufacturer's instruction. Cultures that showed contamination were repeated and excluded from further analysis.

\section{DNA extraction}

DNA was extracted using the FlexiGene DNA Kit (Qiagen, Hilden, Germany) with modifications. Briefly, $500 \mu \mathrm{L}$ of the remaining cell suspension from the process of mycobacterial culture were used. After centrifugation, the sediment was added to $50 \mu \mathrm{L}$ of buffer FG2/QIAGEN protease and vortexed immediately until the pellet was completely homogenized. The mixture was placed in a heating block or water bath and incubated at $65{ }^{\circ} \mathrm{C}$ for $10 \mathrm{~min}$, and then at $95^{\circ} \mathrm{C}$ for $10 \mathrm{~min}$ to stop the enzymatic activity. Fifty microliters of isopropanol (100\%) were added and mixed thoroughly by inversion. Centrifugation at $13,000 \times \mathrm{g}$ for $5 \mathrm{~min}$ was performed and the supernatant was subsequently decanted. After another centrifugation at $13,000 \times \mathrm{g}$ for $5 \mathrm{~min}$ followed by discarding the supernatant, DNA was recovered. The sample was air-dried for at least $5 \mathrm{~min}$. Finally, $30 \mu$ Lof sterile distilled water were added to re-suspend the DNA. The resulting DNA was kept at $-20{ }^{\circ} \mathrm{C}$ for further experiments.

\section{In-house loop-mediated isothermal amplification assay}

A set of LAMP primers, the reaction mixture, and the amplification conditions were as described previously ${ }^{8,9,11}$. Briefly, LAMP reactions were performed in a total volume of $20 \mu \mathrm{L}$ consisting of $30 \mathrm{pmol}$ of each of the inner primers (FIP and BIP), 5 pmol each of the outer primers (F3 and B3), 20 pmol each of the loop primers (FLP and BLP), $1.4 \mathrm{mM}$ 
deoxynucleoside triphosphate, $0.8 \mathrm{M}$ betaine, $20 \mathrm{mM}$ Tris$\mathrm{HCl}(\mathrm{pH} 8.8), 10 \mathrm{mM} \mathrm{KCl}, 10 \mathrm{mM}\left(\mathrm{NH}_{4}\right)_{2} \mathrm{SO}_{4}, 8 \mathrm{mM} \mathrm{MgSO}_{4}$, $8 \mathrm{U}$ Bst DNA polymerase (New England Biolabs, Ipswich, MA, USA) and $1 \mu \mathrm{L}$ of the fluorescent detection reagent (FDR; Eiken Chemical, Tokyo, Japan) with $6 \mu \mathrm{L}$ of extracted DNA. Amplification was performed at $65^{\circ} \mathrm{C}$ for $60 \mathrm{~min}$ in a water bath. Each run contained a positive purified MTB H37Ra DNA $(0.1 \mathrm{ng} / \mathrm{mL})$ control and a negative control (distilled water). LAMP results were examined directly by visual observation. The result was considered positive when the color turned from orange to green. For the amplicon confirmation, $5 \mu \mathrm{L}$ of the LAMP reaction product were analyzed by electrophoresis on $2 \%$ agarose gels.

\section{Xpert MTB/RIF assay}

The Xpert MTB/RIF assay was performed according to the manufacturer's protocol. Briefly, $500 \mu \mathrm{L}$ of cell suspension were mixed with $1.5 \mathrm{~mL}$ of the sample reagent from the test kit. After being vortexed and standing for $15 \mathrm{~min}, 2 \mathrm{~mL}$ of the mixture were transferred into the Xpert MTB/RIF cartridge which then inserted in the module of the GeneXpert machine. The system operated automatically and interpreted the result of the MTB detection and the resistance to rifampicin by measuring the fluorescent signals. The threshold cycle $(\mathrm{Ct})$ of the Xpert MTB/RIF detection in each sample was recorded and analyzed. Invalid results were excluded from further analysis.

\section{Statistical analysis}

Determination of sensitivity, specificity with $95 \%$ confidential interval (CI) and comparison of means were performed using the Student's $t$-test $\left(\mathrm{SAS}^{\circledR}\right.$ University Edition $\left.^{12,13}\right) . P$-values less than 0.05 were considered statistically significant.

\section{RESULTS}

Acid-fast staining and MTB culturing results of clinical specimens

After exclusion of culture contamination and Xpert MTB/RIF invalid results, a total of 204 processed sputum specimens were obtained and analyzed. Of these, 109 were AFB-positive and 95 were AFB-negative by the microscopy of smears. All AFB-positive samples were positive by culture and identified as MTB. For AFB-negative samples, 44 were confirmed as MTB by culture and the other 51 were culture-negative.
Performance of in-house LAMP and Xpert MTB/RIF for MTB detection comparing to acid-fast staining and MTB culturing

All samples were subjected to LAMP and Xpert MTB/ RIF analyses. Of the 204 samples tested, 121 were LAMP positive (color change from orange to bright green) and two were considered positive after the analysis on agarose gels. (given the MTB-positive pattern; Figure 1). Twenty-seven samples were negative by LAMP. For Xpert MTB/RIF, 133 from the 204 samples were considered MTB detected and the others were MTB not detected. The overall sensitivity and specificity of LAMP when compared to MTB culture were $82.4 \%$ (95\% confidential interval [CI]: 75.4-88.0\%) and $100.0 \%$ (95\% CI: $93.0-100.0 \%)$, respectively. When compared with MTB culture, Xpert MTB/RIF showed $86.9 \%$ of sensitivity (95\% CI: $80.5-91.8 \%$ ) and $100.0 \%$ of specificity (95\% CI: $93.0-100.0 \%$ ). Sensitivities of in-house LAMP and Xpert MTB/RIF were 96.3\% (105/109) and $100.0 \%$ (109/109) in smear-positive/culture-positive specimens, and $47.7 \%$ (21/44) and 54.6\% (24/44) in smearnegative/culture-positive specimens. The summary data of the sensitivity analyses were shown in Table 1.

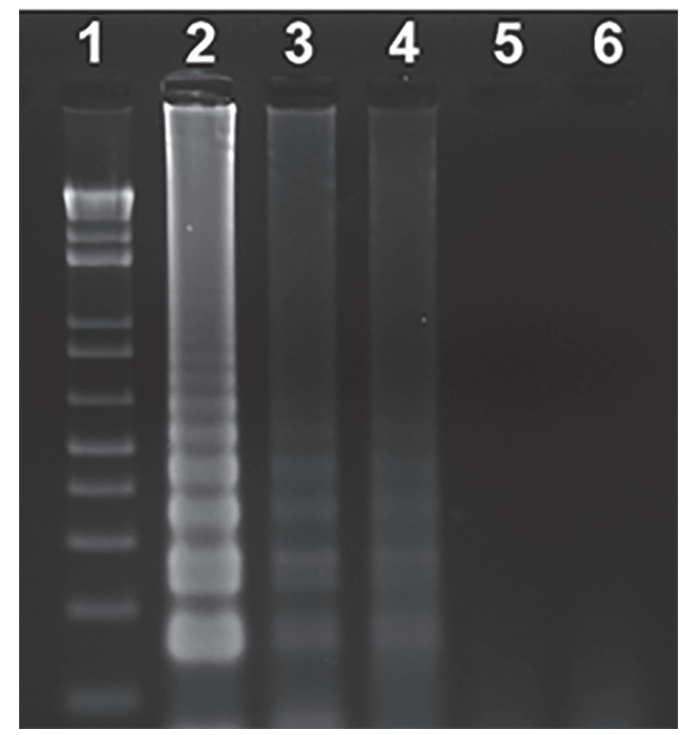

Figure 1 - Gel electrophoresis pattern of the in-house LAMP assay (lane 1- $1 \mathrm{~kb}$ DNA ladder; lane 2- positive control; lane 3-4: negative sample by color change, but positive by gel electrophoresis; lane 5; negative sample by color change and gel electrophoresis; and lane 6- negative control).

\section{Comparison of in-house LAMP and Xpert MTB/RIF for MTB detection}

For the MTB detection, LAMP showed an overall sensitivity and specificity of $94.7 \%$ (95\% CI: 89.5 to 97.9) and $100.0 \%$ (95\% CI: 94.9 to 100.0$)$, respectively, when 
Table 1 - Comparison of an in-house LAMP and the Xpert MTB/RIF results with MTB cultures

\begin{tabular}{|c|c|c|c|c|c|}
\hline \multirow{2}{*}{ Test } & & \multicolumn{2}{|c|}{ MTB culture } & \multirow{2}{*}{$\%$ sensitivity $(95 \% \mathrm{Cl})$} & \multirow{2}{*}{$\%$ specificity $(95 \% \mathrm{Cl})$} \\
\hline & & Positive & Negative & & \\
\hline \multirow{2}{*}{ LAMP } & Positive & $126^{1}$ & 0 & \multirow{2}{*}{82.4 (75.4 to 88.0$)$} & \multirow{2}{*}{$100.0(93.0$ to 100.0$)$} \\
\hline & Negative & $27^{2}$ & 51 & & \\
\hline \multirow{2}{*}{ Xpert MTB/RIF } & MTB detected & $133^{3}$ & 0 & \multirow{2}{*}{86.9 (80.5 to 91.8$)$} & \multirow{2}{*}{100.0 (93.0 to 100.0$)$} \\
\hline & MTB not detected & $20^{4}$ & 51 & & \\
\hline
\end{tabular}

${ }^{1} 105$ were AFB-positive and 21 were negative; ${ }^{2} 4$ were AFB-positive and 23 were negative; ${ }^{3} 109$ were AFB-positive and 24 were negative; ${ }^{4} 20$ were AFB-negative. MTB = Mycobacterium tuberculosis; LAMP = loop-mediated isothermal amplification; $\mathrm{Cl}=$ confidential interval

Table 2 - Comparison of in-house LAMP result with Xpert MTB/RIF

\begin{tabular}{|c|c|c|c|c|c|}
\hline \multirow[b]{2}{*}{ Test } & \multirow[b]{2}{*}{ Result } & \multicolumn{2}{|c|}{ Xpert MTB/RIF } & \multirow[b]{2}{*}{$\%$ sensitivity $(95 \% \mathrm{Cl})$} & \multirow[b]{2}{*}{$\%$ specificity $(95 \% \mathrm{Cl})$} \\
\hline & & MTB detected & $\begin{array}{l}\text { MTB not } \\
\text { detected }\end{array}$ & & \\
\hline \multirow{2}{*}{ LAMP } & Positive & 126 & 0 & \multirow{2}{*}{94.7 (89.5 to 97.9$)$} & \multirow{2}{*}{$100.0(94.9$ to 100.0$)$} \\
\hline & Negative & 7 & 71 & & \\
\hline
\end{tabular}

MTB = Mycobacterium tuberculosis; LAMP = loop-mediated isothermal amplification; $\mathrm{Cl}=$ confidential interval

compared to Xpert MTB/RIF (Table 2). Seven negative cases by LAMP, but positive by Xpert MTB/RIF showed an average threshold cycle $(\mathrm{Ct})$ of 27.0 , while an average of the $\mathrm{Ct}$ from the other 126 positive samples was 18.4. Comparing the average $\mathrm{Ct}$ detected by Xpert MTB/RIF between groups of LAMP-positive and LAMP-negative, a statistically significant difference $(p<0.05)$ was found (Figure 2).

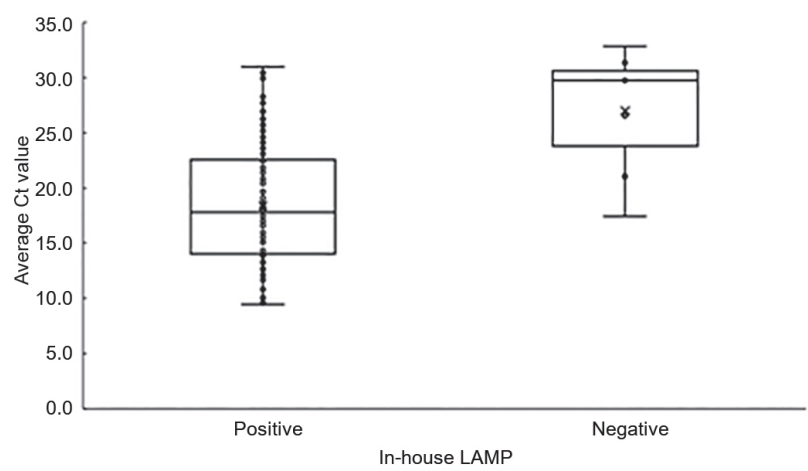

Figure 2 - Distribution of the average Ct values of Xpert MTB/ RIF with respect to the in-house LAMP results. Median of average Ct values for LAMP-positive samples $(n=126)$ and negative samples $(n=7)$ were 17.9 and 29.8, respectively $(p<0.05)$. LAMP, loop-mediated isothermal amplification.

\section{DISCUSSION}

Detection of MTB directly from clinical specimens is a crucial part of TB diagnosis because it helps clinicians to ensure the clinical diagnosis and to reduce the waiting time for the bacterial culture. Acid-fast microscopy remains the main tool for pulmonary tuberculosis diagnosis since it is readily available, rapid and inexpensive. Molecular assays are now selected and used to replace or to be used in combination with the acid-fast staining which exhibits a low sensitivity. In smear-negative cases, the molecular tests have been showing to improve the diagnosis of TB and decrease the time to initiate TB treatment. Xpert MTB/RIF and LAMP are molecular techniques that were recommended by WHO. Because Xpert MTB/RIF is automated, it allows the analysis to be performed in clinics or microscopic centers and it is now widely used in many countries. In contrast, LAMP is a manual cost effective molecular technique proposed to provide an accurate and convenient alternative for TB diagnosis in resource-constrained settings. In the present study, we reported the promising performance of an in-house LAMP targeting MTB $16 S$ rRNA gene to detect MTB from sputum specimens in comparison with the results of microscopy, culture and Xpert MTB/RIF. The overall sensitivity of the in-house LAMP was $82.4 \%$ when compared to the culture, which was lower than in a previous report ${ }^{8}$ and slightly lower than the $84.4 \%$ of a commercial LAMP used in a multicenter study ${ }^{14}$. However, a recent study in Uganda and Vietnam showed that the sensitivity of a commercial LAMP was only 55.4 and $45.5 \%$, respectively ${ }^{15,16}$. The data from a systematic review and meta-analysis, in 2016, showed that any LAMP assays including both commercially available and an in-house, reached sensitivities around $90 \%{ }^{17}$. Indeed, the results of the studies on the performance of techniques can vary greatly according to the prevalence of TB and to the studied populations. Besides differences among LAMP 
protocols, specimen types (direct or processed sputum) and DNA extraction protocols were also the main points resulting in variable values of diagnostic sensitivity among studies. When compared to Xpert MTB/RIF alone, the in-house LAMP showed great sensitivity and specificity, of $94.7 \%$ and $100.0 \%$, respectively. Several studies reported that LAMP and Xpert MTB/RIF had comparably high levels of sensitivity and specificity for the diagnosis of $\mathrm{TB}^{18-20}$. In this comparison, we showed similar sensitivity and specificity of the in-house LAMP and Xpert MTB/RIF. When culture was considered as the gold standard, the sensitivity of LAMP and Xpert were $82.1 \%$ and $86.9 \%$, respectively, and the specificity of both tests was $100 \%$. Therefore, the performance of the inhouse LAMP was promising. This study has also determined the threshold cycle $(\mathrm{Ct})$ of Xpert MTB/RIF detection in LAMP-positive and LAMP-negative groups. The average $\mathrm{Ct}$ value in seven false-negative samples of the in-house LAMP was higher (27.0) compared to that of 18.4 in true-positive cases and the difference was statistically significant. The false-negative results of LAMP in AFB-negative samples might be attributed to the limit of detection of the test. Using culture as the gold standard, the false-negative results were found in 27 samples of the in-house LAMP and in 20 samples of Xpert MTB/RIF which might have occurred because of the lower limit of detection of LAMP and Xpert MTB/RIF compared to that of culture, as described elsewhere ${ }^{3,8,21}$.

The LAMP assay showed a high sensitivity and specificity. The method has other advantages such as easy detection, speed, safety and low cost. Even having several advantages, this simple technique has some disadvantages. Firstly, it is not automated, all the processes (DNA extraction, amplification, and result interpretation) are manual. Secondly, the evaluation in a clinical setting is still limited. Importantly, a major weakness of LAMP compared to Xpert MTB/RIF is that it cannot determine the resistance to rifampicin, hence the use of LAMP in a high rifampicin-resistant burden area is not recommended. In addition, the cost-effectiveness between these two molecular assays also needs to be investigated. Furthermore, the colorimetric determination of the LAMP reaction was easy but might be subjective when determined by visual observation alone ${ }^{22}$, as we found two samples that were negative by visual observation but were positive by agarose gel electrophoresis. However, in general, theses aspects may not be restrictive for the use of LAMP in the rapid TB diagnosis.

One limitation of our study was that the specimens used for DNA extraction and the in-house LAMP and Xpert MTB/RIF analysis were remaining sediments from the decontamination process of the mycobacterial culture. By this approach, we could compare the molecular results with those of culture which remains the gold standard for MTB diagnosis. We introduced the in-house LAMP into the routine practice by using remaining samples. In general, the diagnosis of TB in the hospital is mainly based on smear microscopy, X-ray, and culture confirmation. Xpert MTB/RIF has been recently incorporated into the routine procedures of TB testing in Thailand. However, all specimens cannot be tested by Xpert MTB/RIF due to the high number of cases versus the high cost and the limited supply of cartridges. Also, Xpert MTB/RIF is available only in large hospitals and medical centers or reference laboratories but not in district hospitals. LAMP is an affordable assay for low resource-settings without the need of expensive instruments. Our in-house LAMP can be performed at a much lower cost, around US\$ 1-2, an amount that is similar to the one from a previous report ${ }^{23}$, and compared to the US\$ 9.98 negotiated prices of the Xpert MTB/RIF ${ }^{24}$. In addition, several samples could be handled at the same time and it could provide a shorter turnaround time to results, within 2-3 h. It can be used in hospitals and it is also suitable for use in mycobacterial culture centers in which it would allow the rapid detection of MTB before bacterial cultures. In conclusion, the performance of this in-house LAMP showed a high sensitivity and specificity for rapid MTB detection when compared to culture and Xpert MTB/RIF. It has potential to be provided a rapid, early diagnosis of TB and can be useful for the effective patient management.

\section{ACKNOWLEDGMENTS}

We thank Mae Sot Hospital for providing support and cooperation to do this research.

\section{FUNDING}

This study was supported by the National Institute of Health, Department of Medical Sciences, Ministry of Public Health, Thailand; in part by a grant from the Ministry of Education, Culture, Sports, Science and Technology (MEXT), Japan; for the Joint Research Program of the Research Center for Zoonosis Control, Hokkaido University to Yasuhiko Suzuki; in part by Japan Agency for Medical Research and Development (AMED) under Grant $\mathrm{N}^{\circ}$ JP19fm0108008, and JP18jk0210005 to Yasuhiko Suzuki.

\section{REFERENCES}

1. World Health Organization. Global tuberculosis report 2019. Geneva: WHO; 2019. [cited 2020 Apr 27]. Available from: https://www.who.int/tb/publications/global_report/en/ 
2. World Health Organization. Implementing tuberculosis diagnostics: a policy framework. Geneva: WHO; 2015. [cited 2020 Apr 27]. Available from: https://www.who.int/tb/ publications/implementing_TB_diagnostics/en/

3. Helb D, Jones M, Story E, Boehme C, Wallace E, Ho K, et al. Rapid detection of Mycobacterium tuberculosis and rifampin resistance by use of on-demand, near-patient technology. J Clin Microbiol. 2010;48:229-37.

4. World Health Organization. Automated real-time nucleic acid amplification technology for rapid and simultaneous detection of tuberculosis and rifampicin resistance: Xpert MTB/RIF system: policy statement. Geneva: WHO; 2011. [cited 2020 Apr 27]. Available from: https://www.who.int/tb/publications/ tb-amplificationtechnology-statement/en/

5. Notomi T, Okayama H, Masubuchi H, Yonekawa T, Watanabe $\mathrm{K}$, Amino N, et al. Loop-mediated isothermal amplification of DNA. Nucleic Acids Res. 2000;28:E63.

6. Iwamoto T, Sonobe T, Hayashi K. Loop-mediated isothermal amplification for direct detection of Mycobacterium tuberculosis complex, M. avium, and M. intracellulare in sputum samples. J Clin Microbiol. 2003;41:2616-22.

7. World Health Organization. The use of loop-mediated isothermal amplification (TB-LAMP) for the diagnosis of pulmonary tuberculosis: policy guidance. Geneva: WHO; 2016. [cited 2020 Apr 27]. Available from: https://www.who.int/tb/ publications/lamp-diagnosis-molecular/en/

8. Pandey BD, Poudel A, Yoda T, Tamaru A, Oda N, Fukushima Y, et al. Development of an in-house loop-mediated isothermal amplification (LAMP) assay for detection of Mycobacterium tuberculosis and evaluation in sputum samples of Nepalese patients. J Med Microbiol. 2008;57:439-43.

9. Phetsuksiri B, Rudeeaneksin J, Srisungngam S, Bunchoo S, Klayut W, Sangkitporn S, et al. Loop-mediated isothermal amplification for rapid identification of Mycobacterium tuberculosis in comparison with immunochromatographic SD Bioline MPT64 Rapid ${ }^{\circledR}$ in a high burden setting. Jpn J Infect Dis. 2019;72:112-4.

10. Kent PT, Kubica GP. Public health mycobacteriology: a guide for the level III laboratory. Atlanta: U.S. Department of Health and Human Services, Public Health Service, Centers for Disease Control; 1985.

11. Rudeeaneksin J, Bunchoo S, Srisungngam S, Sawanpanyalert P, Chamnangrom S, Kamolwat A, et al. Rapid identification of Mycobacterium tuberculosis in BACTEC MGIT960 cultures by in-house loop-medicated isothermal amplification. Jpn J Infect Dis. 2012;65:306-11.

12. SAS Institute Inc. The FREQ procedure: sensitivity and specificity. [cited 2020 Apr 27]. Available from: https://documentation. sas.com/?docsetId=procstat\&docsetTarget=procstat_freq details53.htm\&docsetVersion=9.4\&locale $=$ en
13. SAS Institute Inc. MEANS procedure: student's t test. [cited 2020 Apr 27]. Available from: https://documentation.sas. com/?docsetId=proc $\&$ docsetTarget $=$ p 13 qsd0sv8ewi8n 16w0b595383m9.htm\&docsetVersion=9.4\&locale=en

14. Gray CM, Katamba A, Narang P, Girldo J, Zamudio C, Joloba M, et al. Feasibility and operational performance of tuberculosis detection by loop-mediated isothermal amplification platform in decentralized settings: results from a multicenter study. $\mathrm{J}$ Clin Microbiol. 2016;54:1984-91.

15. Nakiyingi L, Nakanwagi P, Briggs J, Agaba T, Mubiru F, Mugenyi M, et al. Performance of loop-mediated isothermal amplification assay in the diagnosis of pulmonary tuberculosis in a high prevalence TB/HIV rural setting in Uganda. BMC Infect Dis. 2018;18:87.

16. Nguyen VA, Nguyen HV, Dinh TV, Du HH, Do CN, Marks GB, et al. Evaluation of Loopamp ${ }^{\mathrm{TM}}$ MTBC detection kit for diagnosis of pulmonary tuberculosis at a peripheral laboratory in a high burden setting. Diagn Microbiol Infect Dis. 2018;90:190-5.

17. Nagai K, Horita N, Yamamoto M, Tsukahara T, Nagakura $\mathrm{H}$, Tashiro K, et al. Diagnostic test accuracy of loopmediated isothermal amplification assay for Mycobacterium tuberculosis: systematic review and meta-analysis. Sci Rep. 2016;6:39090.

18. Yan L, Xiao H, Zhang Q. Systematic review: comparison of Xpert MTB/RIF, LAMP and SAT methods for the diagnosis of pulmonary tuberculosis. Tuberculosis (Edinb). 2016;96:75-86.

19. Yadav R, Sharma N, Khaneja R, Agarwal P, Kanga A, Behera D, et al. Evaluation of the TB-LAMP assay for the rapid diagnosis of pulmonary tuberculosis in Northern India. Int J Tuberc Lung Dis. 2017;21:1150-3.

20. Pham TH, Peter J, Mello FC, Parraga T, Lan NT, Nabeta P, et al. Performance of the TB-LAMP diagnostic assay in reference laboratories: results from a multicentre study. Int J Infect Dis. 2018;68:44-9.

21. Kantor IN, Kim SJ, Frieden T, Laszlo A, Luelmo F, Norval PY, et al. Laboratory services in tuberculosis control: part III: culture. Geneva: WHO; 1998. [cited 2020 Apr 27]. Available from: https://apps.who.int/iris/handle/10665/65942

22. Wong YP, Othman S, Lau YL, Radu S, Chee HY. Loop-mediated isothermal amplification (LAMP): a versatile technique for detection of micro-organisms. J Appl Microbiol. 2018;124:62643.

23. Habeenzu C, Nakajima C, Solo E, Bwalya P, Kajino K, Miller $\mathrm{M}$, et al. Evaluation of in-house loop-mediated isothermal amplification for tuberculosis diagnosis compared with Xpert MTB/RIF. J Infect Dev Ctries. 2017;11:440-4.

24. The Foundation for Innovative New Diagnostics. GeneXpert ${ }^{\circledR}$ : negotiated prices. [cited 2020 Apr 27]. Available from: https:// www.finddx.org/pricing/genexpert 